\title{
Reduction of Potential Risk for Skin Toxicity in Megavoltage Radiotherapy Using a Novel Rigid Couch
}

\author{
MIKOTO TAMURA ${ }^{1}$, HAJIME MONZEN $^{1}$, KENJI MATSUMOTO $^{1}$, \\ MASAHIKO OKUMURA ${ }^{2}$, HIROSHI DOI ${ }^{3}$ and YASUMASA NISHIMURA ${ }^{3}$ \\ ${ }^{1}$ Department of Medical Physics, Graduate School of Medical Science, and \\ ${ }^{3}$ Department of Radiation Oncology, Faculty of Medicine, Kindai University, Osaka-Sayama, Japan; \\ ${ }^{2}$ Department of Central Radiology, Kindai University Hospital, Osaka-Sayama, Japan
}

\begin{abstract}
Aim: In this study, we clarified changes of the surface dose to a low-density material on a carbon couch and verified whether a novel rigid couch (HM couch) could reduce the surface dose. Materials and Methods: We measured the surface dose using only a carbon couch (iBeam Couchtop STANDARD; BrainLab), a low-density material (Styrofoam board) on the carbon couch, and an HM couch for 6 and $10 \mathrm{MV}$ photon beams. Results: A 5-cm styrofoam board placed on the carbon couch reduced the surface dose by approximately 7-9\%, while it had no impact on the depth dose profile; however, in use, such a thickness may cause collision of the patient with the gantry head. The $H M$ couch reduced the surface dose by approximately 7-9\% and shifted the depth dose profile by approximately $0.4 \mathrm{~cm}$ in the depth direction compared to the carbon couch. Conclusion: The HM couch has the potential to reduce skin toxicity and is expected to be useful in clinical practice instead of carbon couches.
\end{abstract}

Many kinds of carbon couches are commonly used in megavoltage radiotherapy to support patients. The carbon fiber composition minimizes imaging artifacts in imageguided radiotherapy (1). Its characteristics are suitable for use with posterior beams (2). However, the surface dose close to the couch tends to be increased, shifting the depth dose curve to the surface of the patient, i.e. the skin $(1,3-6)$. Smith et al. reported a five- to six-fold increase in surface

This article is freely accessible online.

Correspondence to: Hajime Monzen, Ph.D., Department of Medical Physics, Graduate School of Medical Science, Kindai University $377-$ 2, Ohno-Higashi, Osakasayama, Osaka, 589-8511 Japan. Tel: +81 723660221, Fax: +81 723660206, e-mail: hmon@med.kindai.ac.jp

Key Words: Skin toxicity, surface dose, carbon couch, HM couch. dose when using a carbon couch for 6 and $10 \mathrm{MV}$ photon beams, respectively (6). Certain studies have shown that immobilization devices can increase the risk of skin toxicity (7-10). Use of both a carbon couch top and immobilization device was associated with a grade 2 or higher skin toxicity in stereotactic body radiation therapy $(11,12)$.

To solve these problems, the development of the tennis racket table has provided an advantage for reduction of skin toxicity, however, it can exhibit sagging in excess of $5.0 \mathrm{~mm}$, which can cause a systematic error in accuracy and isocentric reproducibility (5). Gray et al. reported that the large air gap created by a patient positioning device reduces the surface dose (13), therefore placing a low-density material on the carbon couch may prevent the creation of air gaps, thereby reducing the surface dose for treatment. An ideal couch material needs to have high permeability and low potential for sagging and should adequately support a patient, allowing the surface dose to be reduced. The HM couch (Toppan Printing Co., Ltd., Tokyo, Japan) has been developed for such a purpose and has the characteristics of being light, strong, rigid, and solid.

In this study, we evaluated the surface doses to a thick low-density material on a carbon couch top and HM couch top to investigate whether the HM couch was able to reduce the surface dose and be useful in clinical application instead of the carbon couch top.

\section{Materials and Methods}

Carbon couch, styrofoam board, and HM couch. We employed a carbon couch (iBeam Couchtop STANDARD, BrainLab, Heimstetten, Germany) $200 \mathrm{~cm}$ long and $53 \mathrm{~cm}$ wide in this study. It was constructed from a plastic foam material with a thickness of $4.6 \mathrm{~cm}$, sandwiched between two layers of carbon fiber, each with a thickness of $0.2 \mathrm{~cm}$. Its maximum load was $185 \mathrm{~kg}$ (14). Styrofoam board (E-Board C; ESFORM, Nagano, Japan, 1.5, 3.0, and $5.0 \mathrm{~cm}$ thick) was employed as a low-density material. The HM couch (Toppan Printing Co., Ltd., Tokyo, Japan) is shown in Figure 

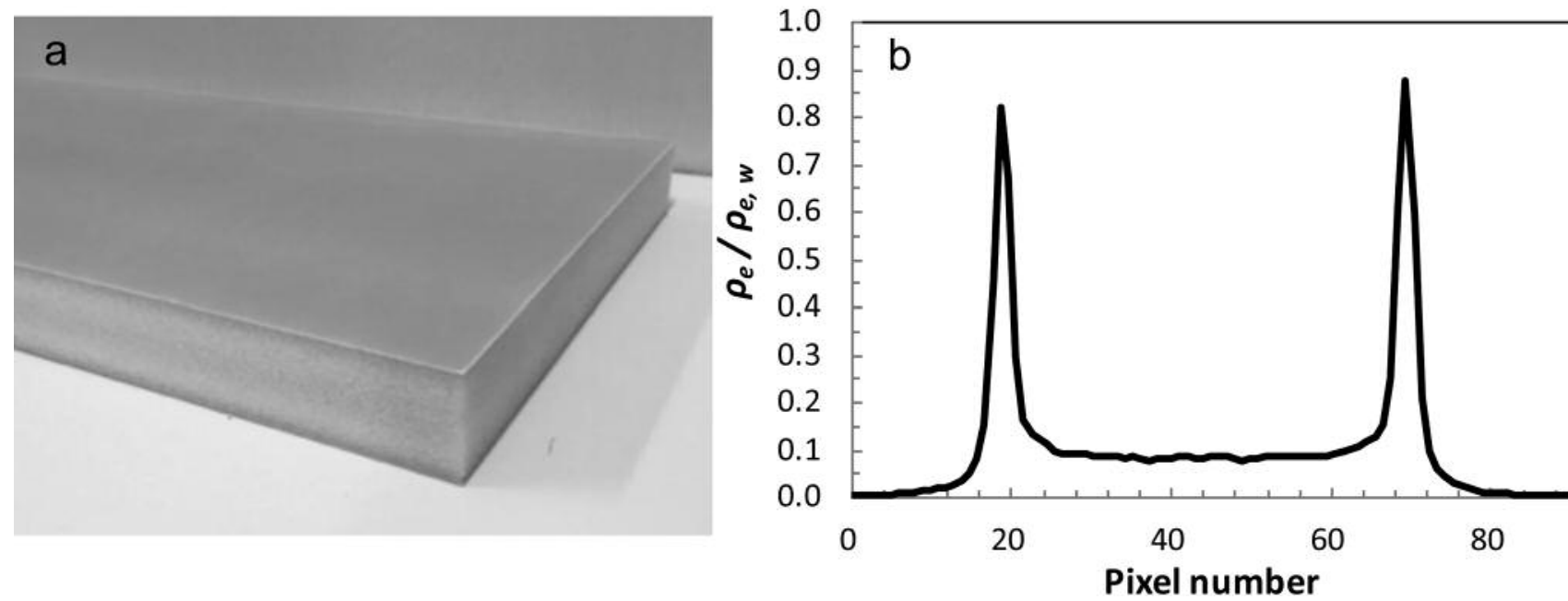

Figure 1. Photograph of HM couch (a) and relative water electron density profile (b). The pixel size was 0.976 mm. The HM couch is composed of thin glass fiber sandwiching a polycarbonate foam core with a total thickness of $5.0 \mathrm{~cm}$.

1a. It is constructed from polycarbonate foam sandwiched between two thin layers of glass fiber, measuring $5.0 \mathrm{~cm}$ in thickness. The polycarbonate is very light and has one of the highest weight resistances of plastics, and a density of $0.1 \mathrm{~g} / \mathrm{cm}^{3}$. The components of the glass fiber ( $\mathrm{wt} \%$ ) was $53.0 \% \mathrm{SiO}_{2}, 15.0 \% \mathrm{Al}_{2} \mathrm{O}_{3}, 21.0 \% \mathrm{CaO}$, $2.0 \% \mathrm{MgO}, 8.0 \% \mathrm{~B}_{2} \mathrm{O}_{3}$, and $0.3 \% \mathrm{Na}_{2} \mathrm{O}$ and $\mathrm{K}_{2} \mathrm{O}$, and with density of $2.55 \mathrm{~g} / \mathrm{cm}^{3}$.

To assess the structure of the HM couch, computed tomography (CT) (Optima, GE Healthcare, Little Chalfont, UK) was employed, using a section thickness of $0.2 \mathrm{~cm}, \mathrm{FOV}=50 \mathrm{~cm}$, and tube voltage of $120 \mathrm{kV}$. The relative electron densities were calculated from CT values and the electron density table in the treatment planning system. Figure $1 \mathrm{~b}$ shows the $\mathrm{CT}$ images and the relative electron density profile for the HM couch. Additionally, we performed a load test on the HM couch to verify its load capacity of $185 \mathrm{~kg}$ (the same as that for the carbon couch). Figure 2 shows the schema of geometry for the load test. The size of the HM couch was $200 \times 53 \times 5 \mathrm{~cm}$. Figure $2 \mathrm{a}$ shows the photograph of a broken HM couch. The load power was $2 \mathrm{~N}$ as shown in Figure 2b. Figure $2 \mathrm{c}$ shows the result of the load test. The maximum load of the HM couch, $2 N$, was $5.88 \mathrm{kN}(600 \mathrm{~kg})$. Therefore, the HM couch can hold a large load adequately.

Surface dose measurements. We used a solid water phantom (Gammex RMI, Miccleton WI, USA), and a plane parallel ionization chamber (PPIC) (Murkus Ion Chamber; PTW, Freiburg, Germany) to measure the absorbed surface doses for 6 and $10 \mathrm{MV}$ photon beams from a clinical linear accelerator (TrueBeam; Varian Medical Systems, Palo Alto CA, USA). The dose of 200 MU was delivered with a field size of $10 \times 10 \mathrm{~cm}^{2}$ at source to surface distance (SSD) of $100 \mathrm{~cm}$.

In measurements with the PPIC, Figure 3 shows the experimental geometry for measurements of surface doses with the carbon couch (Figure 3a) and with the styrofoam board on the carbon couch (Figure 3b). Firstly, we measured the surface doses without the carbon couch at a gantry angle of $0^{\circ}$. Then we measured the surface doses for the carbon couch (Figure 3a) and styrofoam board of 1.5,
3.0 , and $5.0 \mathrm{~cm}$ thickness on the carbon couch (Figure 3b). Figure $3 \mathrm{c}$ shows the schema of geometry for measurements of surface doses for the HM couch. We compared the mean surface dose from three irradiations for the surface dose measurements.

In the surface dose measurements, a charged particle equilibrium was not established because the ionization chamber was located in the build-up region, which can cause perturbation effects in PPIC by scattered radiation, mostly from the chamber sidewall (15). The perturbation effects cause overestimation of surface dose (16). Therefore, we measured the depth of maximum dose $\left(d_{\max }\right)$ for 6 and $10 \mathrm{MV}$ photon beams and set them as reference doses, and corrected the perturbation effects (15).

Depth dose profiles for carbon couch, styrofoam board on the carbon couch, and HM couch. We obtained the depth dose profiles with the carbon couch, styrofoam board of each thickness $(1.5,3.0$, and $5.0 \mathrm{~cm}$ ) on the carbon couch, and HM couches as shown in Figure $3 \mathrm{a}, \mathrm{b}$, and $\mathrm{c}$, respectively, using the solid water phantom and PPIC for 6 and $10 \mathrm{MV}$ photon beams. Measurements were taken with $100 \mathrm{MU}$ irradiation with a field size of $10 \times 10 \mathrm{~cm}^{2}$ at an SSD of $100 \mathrm{~cm}$. The depth dose profiles were normalized to the doses at a depth of $5.0 \mathrm{~cm}$ in the solid water phantom in which a charged particle equilibrium was established. In the build-up region, the doses were corrected for perturbation effect (15).

\section{Results}

Surface dose. Table I shows the surface doses and the values of $d_{\max }$ with and without the carbon couch, and styrofoam board on the carbon couch and HM couch for the 6 and 10 MV photon beams. The standard deviations of measurements by PPIC were within $0.5 \%$. The surface doses for the $5.0 \mathrm{~cm}$ styrofoam board on the carbon couch and HM couch were approximately $7-9 \%$ less than that for the carbon couch alone. 
a

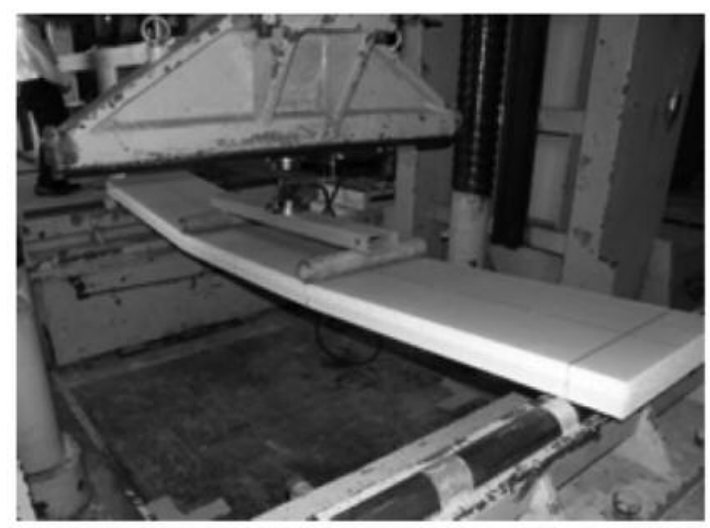

b
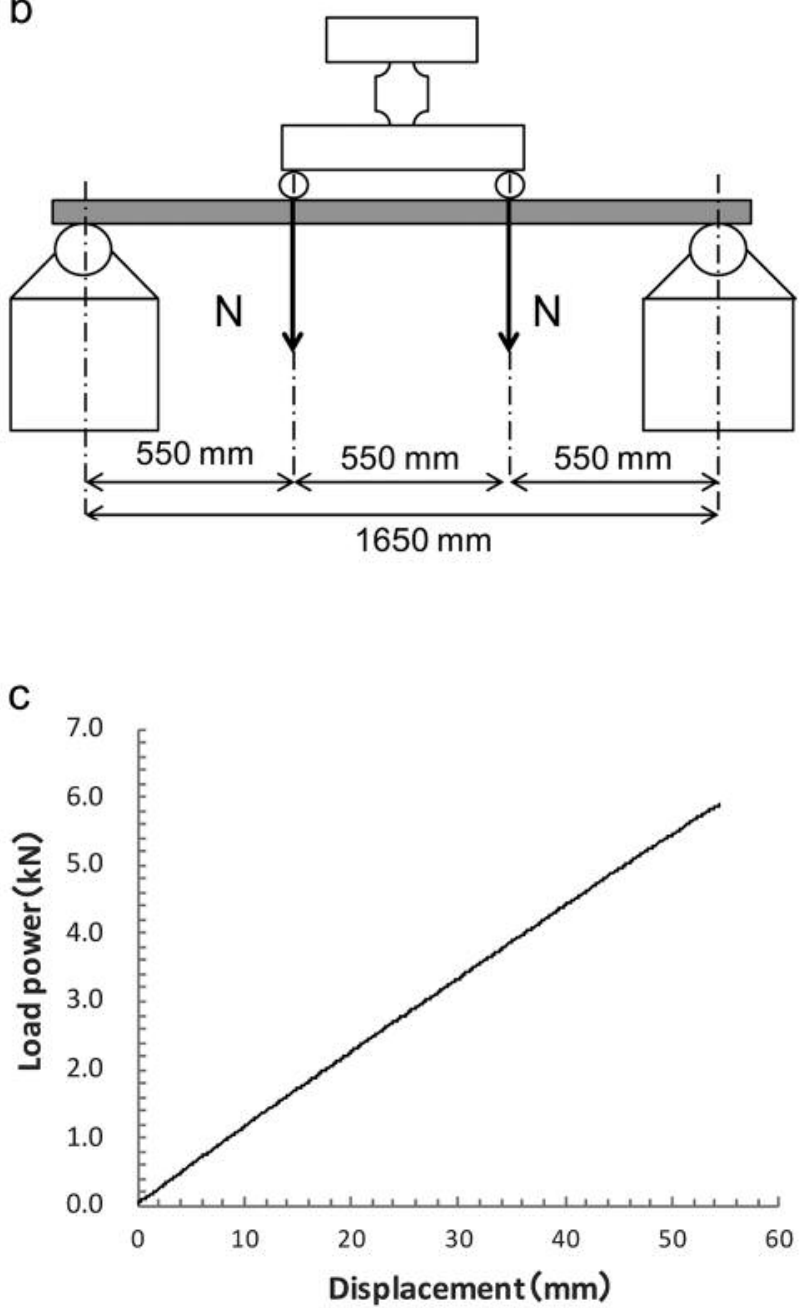

Figure 2. Photograph (a) and schema (b) of the geometry for the load test of the HM couch, and the results of the test (c). The maximum load value of the HM couch, defined as the breaking point (shown in the photograph), $2 \mathrm{~N}$, was $5.88 \mathrm{kN}$ (600 kg).
Depth dose profiles for carbon couch, styrofoam board on the carbon couch, and HM couch. Figure 4 shows the depth dose profiles for the styrofoam boards of different thicknesses on the carbon couch for the 6 and $10 \mathrm{MV}$ photon beams. The values of $d_{\max }$ were almost equal without and with styrofoam boards, while the relative surface doses decreased as the thickness of the styrofoam board increased. With the $6 \mathrm{MV}$ photon beams, the reduction in surface dose compared to the carbon couch alone were $0.0 \%, 1.3 \%$, and $5.1 \%$ for styrofoam boards of 1.5, 3.0, and $5.0 \mathrm{~cm}$ thickness, respectively (Figure 4a). For the $10 \mathrm{MV}$ photon beams, the corresponding reduction of surface dose compared with the carbon couch alone were $2.1 \%, 4.3 \%$, and $8.0 \%$, respectively (Figure $4 \mathrm{~b}$ ).

Figure 5 shows the depth dose profiles for the carbon couch and HM couch with the 6 and $10 \mathrm{MV}$ photon beams. The HM couch reduced the surface dose by $7.9 \%$ and shifted the $d_{\max }$ value $0.4 \mathrm{~cm}$ in the depth direction compared to the carbon couch for $6 \mathrm{MV}$ photon beams (Figure 5a). For the $10 \mathrm{MV}$ photon beams, the HM couch reduced the surface dose by $9.9 \%$ and shifted the $d_{\max }$ value $0.4 \mathrm{~cm}$ in the depth direction compared to the carbon couch (Figure 5b). The surface doses for the HM couch were less than those using the $5.0 \mathrm{~cm}$ styrofoam board on the carbon couch by approximately $2-3 \%$. In the region where charged particle equilibrium was established, the gradients of depth dose profiles for the carbon couch and HM couch were equal for both the 6 and $10 \mathrm{MV}$ photon beams. The depth dose profiles for the HM couch were shifted approximately $0.4 \mathrm{~cm}$ in the depth direction compared to the carbon couch, which indicates the water-equivalent thickness of the HM couch was approximately $0.4 \mathrm{~cm}$ less than that of the carbon couch.

\section{Discussion}

In this study, we investigated the surface doses for a carbon couch and styrofoam board as a low-density material on a carbon couch. The surface dose with carbon couch was increased fourto five-fold compared to without that of a carbon couch alone since the carbon couch shifted the depth dose profile to the surface by approximately $1.2 \mathrm{~cm}$ (Table I), that accorded the nominal value of water-equivalent thickness. However, the styrofoam board, especially of $5.0 \mathrm{~cm}$ thickness, reduced the surface dose as shown in Figure 4, since build-down might be caused in the styrofoam board and secondary build-up was established (13). The combination of both a material of low absorption and a large gap was effective in reducing the surface dose. However, the large gap of $5.0 \mathrm{~cm}$ might not allow its use in clinical practice due to the increased possibility of a collision between the patient and the gantry head.

We also verified the dosimetric characteristics for the HM couch. Used instead of the carbon couch, the HM couch reduced the surface dose by an additional 7-9\% (Table I), since the depth dose profile for HM couch was shifted from the 
a

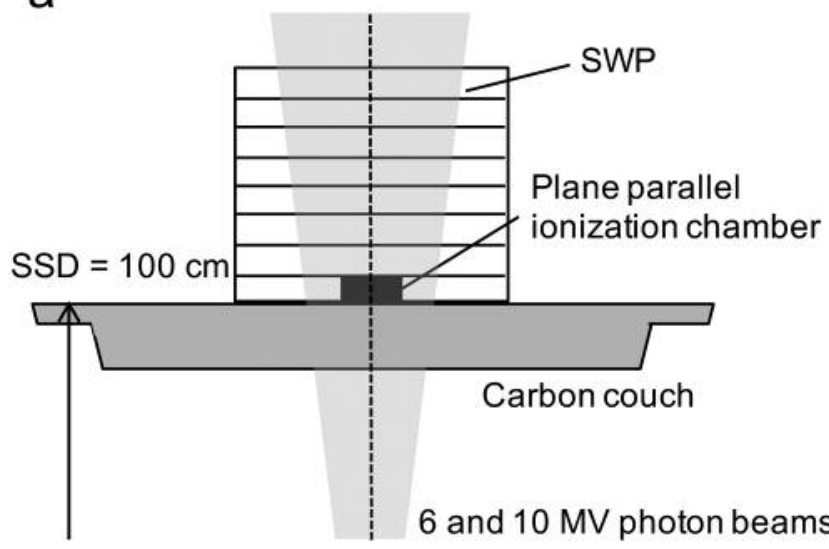

b

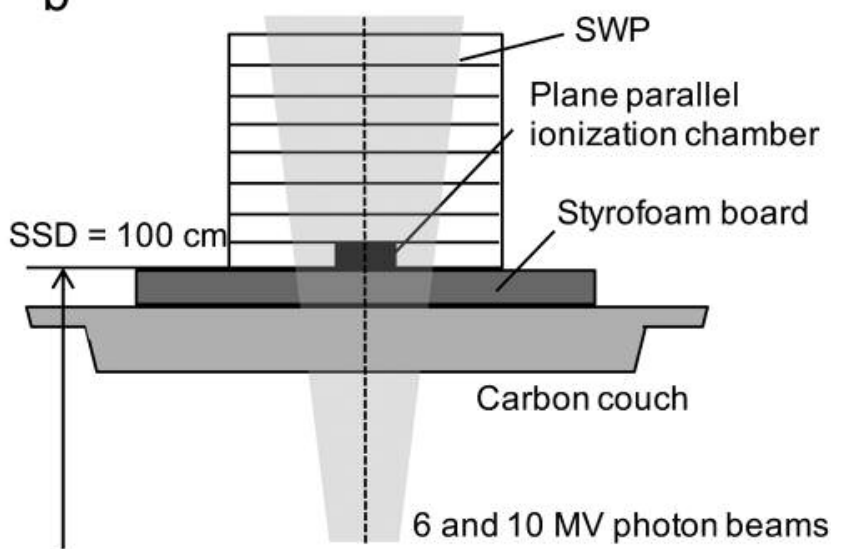

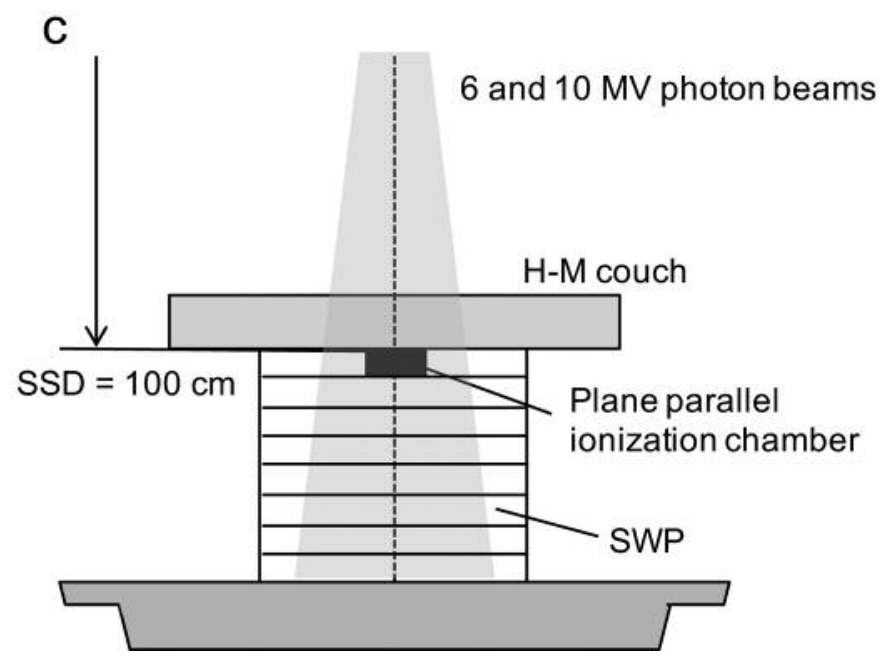

Figure 3. Schematic views of measurements of absorbed surface doses and depth dose profiles for carbon couch (a), carbon couch with styrofoam boards of 1.5, 3.0 or $5.0 \mathrm{~cm}$ thicknesses (b), and HM couch (c) using a plane parallel ionization chamber. SSD: Source to surface distance; SWP: solid water phantom.

Table I. The surface doses and depths of maximum dose $\left(d_{\max }\right)$ without carbon couch, with carbon couch, carbon couch with $1.5,3.0$, and $5.0 \mathrm{~cm}$ styrofoam board, and HM couch.

\begin{tabular}{|c|c|c|c|c|}
\hline & \multicolumn{2}{|c|}{$6 \mathrm{MV}$} & \multicolumn{2}{|c|}{$10 \mathrm{MV}$} \\
\hline & Surface dose (Gy) & $d_{\max }(\mathrm{cm})$ & Surface dose (Gy) & $d_{\max }(\mathrm{cm})$ \\
\hline Without carbon couch & 0.431 & 1.5 & 0.303 & 2.4 \\
\hline \multicolumn{5}{|l|}{ Carbon couch } \\
\hline Alone & 1.721 & 0.3 & 1.586 & 1.2 \\
\hline With $1.5 \mathrm{~cm}$ Styrofoam board & 1.740 & 0.2 & 1.554 & 1.2 \\
\hline With $3.0 \mathrm{~cm}$ Styrofoam board & 1.714 & 0.3 & 1.515 & 1.2 \\
\hline With $5.0 \mathrm{~cm}$ Styrofoam board & 1.585 & 0.3 & 1.458 & 1.3 \\
\hline With HM couch & 1.598 & 0.7 & 1.444 & 1.6 \\
\hline
\end{tabular}


a 6 MV Photon beams

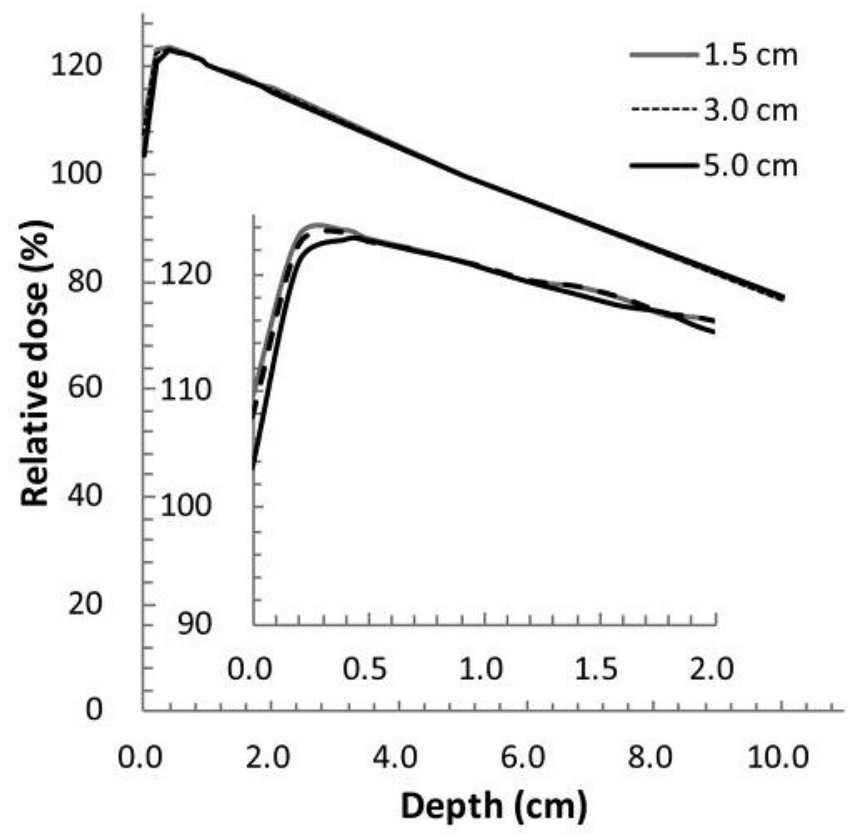

b $10 \mathrm{MV}$ Photon beams

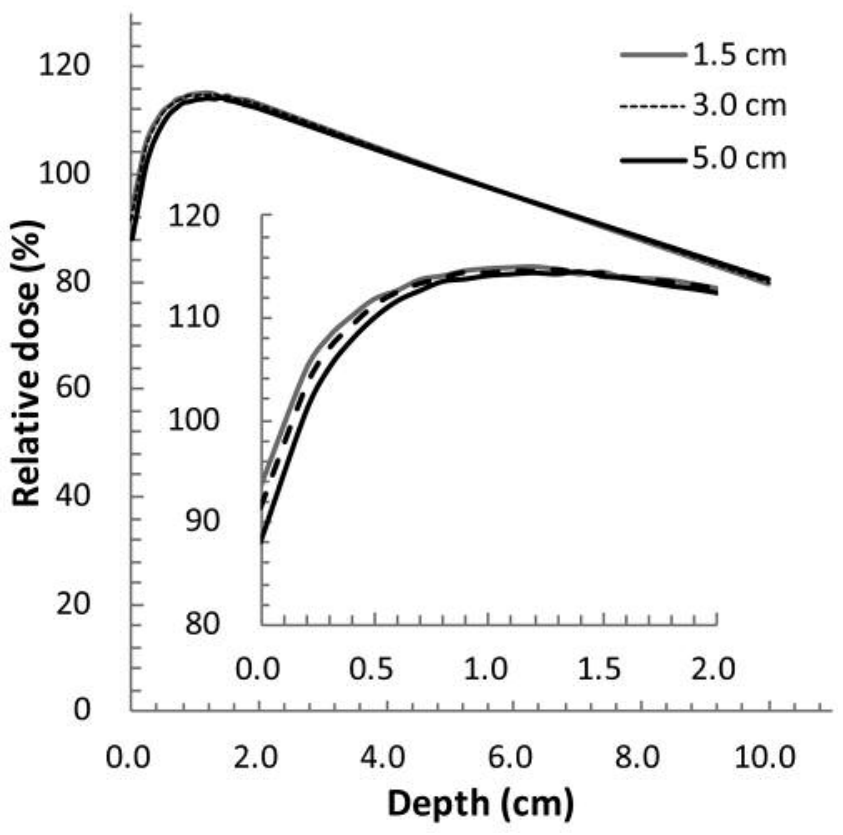

Figure 4. Depth dose profiles for the carbon couch with styrofoam boards with thicknesses of 1.5, 3.0, and $5.0 \mathrm{~cm}$ for 6 (a) and 10 (b) MV photon beams, which were normalized to the dose at a depth of $5.0 \mathrm{~cm}$ in a solid water phantom.

a 6 MV Photon beams

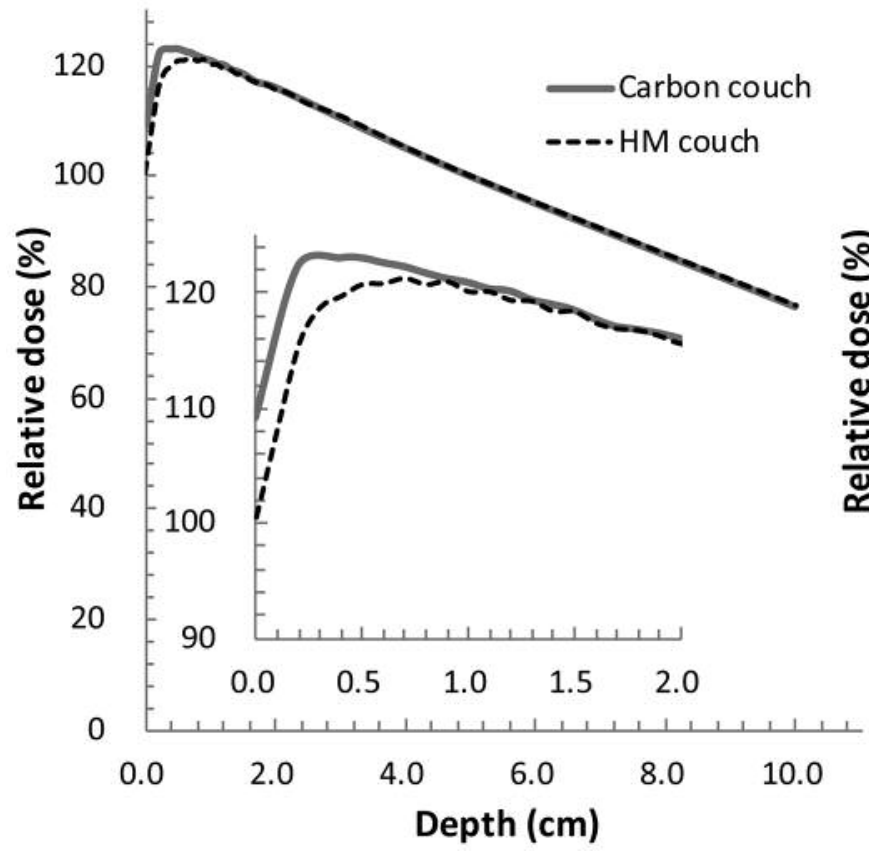

b $10 \mathrm{MV}$ Photon beams

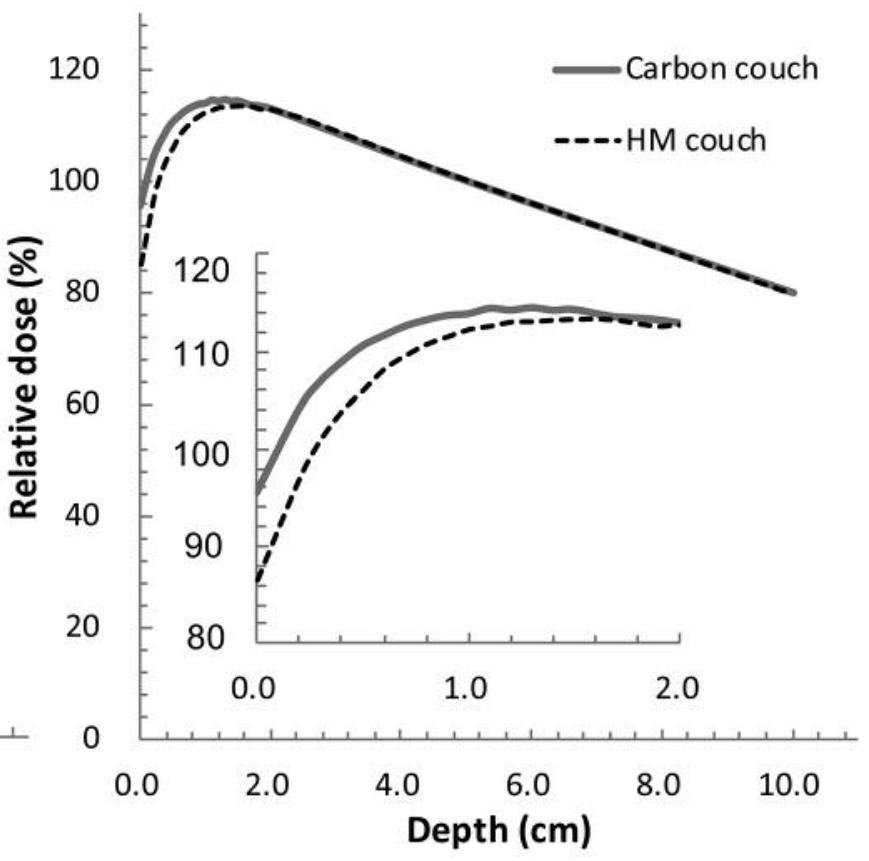

Figure 5. Depth dose profiles for the carbon couch and HM couch with 6 (a) and 10 (b) MV photon beams, which were normalized to the doses at a depth of $5.0 \mathrm{~cm}$ in a solid water phantom. 
surface (Figure 5). The differences in water-equivalent thickness for the carbon couch and HM couch caused this shift of depth dose profile. The water-equivalent thickness of HM couch was approximately $0.76 \pm 0.02 \mathrm{~cm}$, as determined from the relative electron density profiles shown in Figure 1b (17), while that of the carbon couch was approximately $1.2 \mathrm{~cm}$. The surface dose for the HM couch was almost equal to that of the $5.0 \mathrm{~cm}$ styrofoam board on the carbon couch where the irradiation MU was equal. However, in the depth dose profile normalized at an arbitrary depth at which a charged particle equilibrium was established, the surface dose for the HM couch was approximately $2-3 \%$ less than that of the $5.0 \mathrm{~cm}$ styrofoam board on the carbon couch since the HM couch had the characteristic of low attenuation for radiation compared with the $5.0 \mathrm{~cm}$ styrofoam board on the carbon couch. This indicates that the lower irradiation rate was enough to deliver a prescribed dose using the HM couch compared with the $5.0 \mathrm{~cm}$ styrofoam board on the carbon couch. A low irradiation rate is particularly more important for stereotactic body radiation therapy, which can be associated with significant skin toxicity (11).

Some reports have described methods for reduction of skin toxicity. The accurate calculation of skin dose with radiation treatment planning is important for predicting the influence of the carbon couch $(3,5,6,9)$ and another recommendation is the use of multiple beams (11). The HM couch appears to reduce the surface dose associated with skin toxicity more simply than other methods. Its lower cost also recommends its use instead of the carbon couch.

\section{Conclusion}

Skin toxicity can be reduced by placing a low-density material of $5.0 \mathrm{~cm}$ or thicker on the carbon couch, although it may cause a collision between the patient and gantry head. The HM couch has also the potential to reduce skin toxicity to the same extent with reduction of the likelihood of collision. The HM couch is expected to be useful in clinical practice instead of carbon couches.

\section{Conflicts of Interest}

Hajime Monzen has a consultancy agreement with, and financial interest in, TOPPAN PRINTING CO., LTD, Tokyo.

\section{Acknowledgements}

This work was supported by the JSPS KAKENHI [grant numbers 17K09071 and 16K09027]. We would like to thank Mr. Masaru Hayakawa for his valuable support.

\section{References}

1 Sepälä JKH and Kulmala JAJ: Increased beam attenuation and surface dose by different couch inserts of treatment tables used in megavoltage radiotherapy. J Appl Clin Med Phys 12: 15-23, 2011.
2 McCormack S, Diffey J and Morgan A: The effect of gantry angle on megavoltage photon beam attenuation by a carbon fiber couch insert. Med Phys 32: 483-487, 2005.

3 Meydanci TP and Kemikler G: Effect of a carbon fiber tabletop on the surface dose and attenuation for high-energy photon beams. Radiat Med 26: 539-544, 2008.

4 Spezi E and Ferri A: Dosimetric characteristics of the SIEMENS IGRT carbon fiber tabletop. Med Dosim 32: 295-298, 2007.

5 Higgins DM, Whitehurst P and Morgan AM: The effect of carbon fiber couch inserts on surface dose with beam size variation. Med Dosim 26: 251-254, 2001.

6 Smith DW, Christophides D, Dean C, Naisbit M, Mason J and Morgan A: Dosimetric characterization of the iBEAM evo carbon fiber couch for radiotherapy. Med Phys 37: 3595-3606, 2010.

7 Lee KW, Wu JK, Jeng SC, Hsueh YW and Cheng JCH: Skin dose impact from vacuum immobilization device and carbon fiber couch in intensity-modulated radiation therapy for prostate cancer. Med Dosim 34: 228-232, 2009.

8 Vieira SC, Kaarten RSJP, Dirkx MLP and Heijmen BJM: Twodimensional measurement of photon beam attenuation by the treatment couch and immobilization devices using an electronic portal imaging device. Med Phys 30: 2981-2987, 2003.

9 Lee N, Chuang C, Quivey JM, Phillipse TL, Akazawa P, Verhey LJ and Xia P: Skin toxicity due to intensity-modulated radiotherapy for head-and-neck carcinoma. Int J Radiat Oncol Biol Phys 53: 630-637, 2002.

10 Carl $\mathrm{J}$ and Vestergaard A: Skin damage probabilities using fixation materials in high-energy photon beams. Radiother Oncol 55: 191-198, 2000.

11 Hoppe BS, Laser B, Kowalski AV, Fontenla SC, Pena-Greenberg E, Yorke ED, Lovelock DM, Hunt MA and Rosenzweig KE: Acute skin toxicity following stereotactic body radiation therapy for stage I non-small-cell lung cancer: Who at risk? Int J Radiat Oncol Biol Phys 72: 1281-1286, 2008.

12 Arthur JO, Lee G, Heng L, Ivaylo M and Andrew M: Dosimetric effects caused by couch tops and immobilization devices: Report of AAPM Task Group 176. Med Phys 41: 061501, 2014.

13 Gray A, Oliver LD and Johnston PN: The accuracy of the pencil beam convolution and anisotropic analytical algorithms in predicting the dose effects due to attenuation from immobilization devices and large air gap. Med Phys 36: 3181-3191, 2009.

14 Njeh CF, Parker J, Spurgin J and Rhoe E: A validation of carbon fiber imaging couch top modeling in two radiation therapy treatment planning systems: Philips Pinnacle3 and BrainLAB iPlan RT Dose. Radiat Oncol 7: 190-200, 2012.

15 Gerbi BJ and Khan FM: Measurement of dose in the buildup region using fixed-separation plane-parallel ionization chambers. Med Phys 17: 17-26, 1990.

16 Das IJ, Cheng CW, Watts RJ, Ahnesjö A, Gibbons J, Li XA, Lowenstein J, Mitra RK, Simon WE and Zhu TC: Accelerator beam data commissioning equipment and procedures: Report of the TG-106 of the Therapy Physics Committee of the AAPM. Med Phys 35: 4186-4215, 2014.

17 Gerig LH, Niedbala M and Nyiri BJ: Dose perturbations by two carbon fiber treatment couches and the ability of a commercial treatment planning system to predict these effects. Med Phys 37: 322-328, 2010

Received January 31, 2018

Revised February 23, 2018

Accepted February 26, 2018 